\title{
Discovery of gene expression-based pharmacodynamic biomarker for a p53 context-specific anti-tumor drug Weel inhibitor Shinji Mizuarai ${ }^{1}$, Kazunori Yamanaka1 ${ }^{1}$, Hiraku Itadani ${ }^{1}$, Tsuyoshi Arai ${ }^{1}$, Toshihide Nishibata ${ }^{1}$, Hiroshi Hirai ${ }^{1}$ and Hidehito Kotani*1,2
} \author{
300-2611, Japan and 2Corporate services, Banyu Pharmaceutical Co, Ltd, Chiyoda-Ku, Tokyo 102-8667, Japan \\ Email: Shinji Mizuarai - shinji_mizuarai@merck.com; Kazunori Yamanaka - kazunori_yamanaka@merck.com \\ Hiraku Itadani - hiraku_itadani@merck.com; Tsuyoshi Arai - tsuyoshi_arai@merck.com; \\ Toshihide Nishibata - toshihide_nishibata@merck.com; Hiroshi Hirai - hiroshi_hirai@merck.com; \\ Hidehito Kotani* - hidehito_kotani@merck.com \\ * Corresponding author
}

Address: ${ }^{1}$ Department of Oncology, Tsukuba Research Institute, Merck Research Laboratories, Banyu Pharmaceutical Co, Ltd, Tsukuba, Ibaraki

Published: 8 June 2009

Molecular Cancer 2009, 8:34 doi:10.1/86/1476-4598-8-34
Received: 13 April 2009

Accepted: 8 June 2009

This article is available from: http://www.molecular-cancer.com/content/8/1/34

(c) 2009 Mizuarai et al; licensee BioMed Central Ltd.

This is an Open Access article distributed under the terms of the Creative Commons Attribution License (http://creativecommons.org/licenses/by/2.0), which permits unrestricted use, distribution, and reproduction in any medium, provided the original work is properly cited.

\begin{abstract}
Background: Weel is a tyrosine kinase regulating S-G2 cell cycle transition through the inactivating phosphorylation of $C D C 2$. The inhibition of Weel kinase by a selective small molecule inhibitor significantly enhances the anti-tumor efficacy of DNA damaging agents, specifically in p53 negative tumors by abrogating S-G2 checkpoints, while normal cells with wild-type $p 53$ are not severely damaged due to the intact function of the GI checkpoint mediated by $p 53$. Since the measurement of mRNA expression requires a very small amount of biopsy tissue and is highly quantitative, the development of a pharmacodynamic (PD) biomarker leveraging mRNA expression is eagerly anticipated in order to estimate target engagement of anti-cancer agents.
\end{abstract}

Results: In order to find the Weel inhibition signature, mRNA expression profiling was first performed in both $\mathrm{p} 53$ positive and negative cancer cell lines treated with gemcitabine and a Weel inhibitor, MK-1775. We next carried out mRNA expression profiling of skin samples derived from xenograft models treated with the Weel inhibitor to identify a Weel inhibitor-regulatory gene set. Then, the genes that were commonly modulated in both cancer cell lines and rat skin samples were extracted as a Weel inhibition signature that could potentially be used as a PD biomarker independent of p53 status. The expression of the Weel inhibition signature was found to be regulated in a dose-dependent manner by the Weel inhibitor, and was significantly correlated with the inhibition level of a direct substrate, phosphorylated-CDC2. Individual genes in this Weel inhibition signature are known to regulate S-G2 cell cycle progression or checkpoints, which is consistent with the mode-of-action of the Weel inhibitor.

Conclusion: We report here the identification of an mRNA gene signature that was specifically changed by gemcitabine and Weel inhibitor combination treatment by molecular profiling. Given the common regulation of expression in both xenograft tumors and animal skin samples, the data suggest that the Weel inhibition gene signature might be utilized as a quantitative PD biomarker in both tumors and surrogate tissues, such as skin and hair follicles, in human clinical trials. 


\section{Background}

A diversity of anti-tumor agents is known to cause DNA damage resulting in the activation of $\mathrm{G} 1$ and $\mathrm{G} 2$ cell cycle checkpoints [1-3]. Normal somatic cells with functional p53 arrest the cell cycle both at G1 and G2 phases by transactivating p53 regulatory genes upon DNA damage $[4,5]$. However, the G1 checkpoint is frequently compromised in multiple types of cancers due to loss-of-function mutations in the p53 gene [6,7]. Cancer cells with dysfunctional p53 are more reliant on the G2 checkpoint in order to repair damaged DNA. Wee1 kinase, which acts as a critical driver of G2-M cell cycle progression, is involved in S-G2 checkpoints through inactivating phosphorylation of CDC2 at the Y15 residue [8,9]. When DNA is damaged in cells, Wee1 is phosphorylated at S549 by several kinases, including CHEK1, followed by binding to 14-3-3 proteins which leads to stabilization of the Wee1 protein [10-12]. The phosphorylated and stabilized Wee1 increases the level of inactivated phoshorylated-CDC2, preventing the damaged cells from entering into premature mitosis without repairing the DNA. Although the activation mechanism is still controversial, various studies have established the essential function of Wee1 in the regulation of S-G2 cell cycle arrest in response to DNA damage.

Given the pivotal role of Wee1 in the S-G2 checkpoint, the inhibition of Wee1 kinase is expected to exert an antitumor effect by abrogating the G2 checkpoint, specifically in 553 negative tumors in combination with DNA damaging drugs. Several previous studies have illustrated the p53-context dependent anti-tumor efficacy of Wee1 inhibition in vitro [13-15]. A potent Wee1 inhibitor, PD0166283, sensitizes p53-negative cancer cells to radiation-induced cell death compared with p53-positive cells $[13,14]$. It was also shown that Wee1 silencing by siRNA potentiates the anti-tumor effect of Adriamycin in p53defective HeLa cells, although normal mammary epithelial cells with wild-type p53 are not severely damaged [15]. Recently, we have developed a new class of small molecule Wee1 inhibitor as a G2 checkpoint abrogator, MK-1775 [16]. The Wee1 inhibitor induces cell death selectively in p53-negative cells compared with isogenic p53-positive cells in combination with DNA damaging agents such as gemcitabine, carboplatin, and cisplatin. The assessment of the primary substrate, phospho-CDC2, ensured that the p53 context-specificity was mediated by Wee1 inhibition. We also demonstrated that significant sensitization to various DNA damaging agents is observed in p53 negative xenograft tumors in rodents, providing the initial evidence that Weel inhibition enhances the effect of standard care medicine in vivo via abrogating the G2 checkpoint. Clinical development of the Wee1 inhibitor as a p53 context-specific sensitizer would potentially improve the low therapeutic indices and narrow therapeu- tic window from which current chemotherapeutic agents are suffering.

Development of pharmacodynamic (PD) biomarkers is critically important in cancer drug development in order to examine whether drugs are modulating the intended therapeutic targets or pathways [17-19]. Conventionally, immunohistochemistry (IHC) assays for protein-biomarkers have played an important role in assessing the target engagement level of drugs; such biomarkers include phosphorylated-EGFR for Iressa [20], and phosphorylatedCRKL for Gleevec [21]. For the Wee1 inhibitor, the phosphorylation level of CDC2 is a promising PD biomarker since it is a primary substrate for Wee1 kinase [14-16]. Indeed, reduction of phosphorylated-CDC2 at Tyr15 has been observed in both in vitro and in vivo studies, confirming that Wee1 inhibitors were engaging the target. Furthermore, the level of phosphorylation at Y15 is correlated with the anti-tumor efficacy of the Wee1 inhibitor. However, IHC assays for protein biomarkers have presented several challenges when developed in a clinical setting. First, IHC markers require a relatively large amount of biopsy tissue and morphological integrity, and these requirements are difficult to fulfill for some tumor biopsy methods, such as fine needle aspiration [22]. Second, IHC assays for proteins are not quantitative, since the expression level is usually indicated by the intensity scores of chromogens ranging from 0 to 3, which is a relatively arbitrary index. The development of mRNA gene expression signatures for anticancer drugs is an intriguing approach to overcome these drawbacks, since the measurement of mRNA requires smaller amounts of biopsy samples, and is highly quantitative when measured with an RT-qPCR assay. Multiple previous studies have measured mRNA expressions as PD gene biomarkers for estimating target engagement or predicting early response of anti-cancer agents such as KDR [23], COXII [24], or histone deacetylase inhibitors [25], providing evidence that mRNA gene signatures are suitable to quantitatively represent the indices.

The purpose of the present study was to develop a Wee1 inhibition gene signature measuring the change in expression caused by a combination treatment of Wee 1 inhibitor and gemcitabine. Genome-wide gene expression in both cancer cells and skin tissues was analyzed to find a Wee1 gene signature that can be utilized in both tumor and surrogate tissues. The availability of the Weel gene signature in skin samples offers an advantage due to the difficulty of obtaining tumor biopsies from patients. In addition, dose-dependent expression changes of the Wee1 gene signature in rodent xenograft tumors and skin samples were correlated with the level of phosphorylatedCDC2 and anti-tumor efficacy of the Wee1 inhibitor. The expression pattern and function of the Weel gene signa- 
ture are consistent with mode of action of the Wee 1 inhibitor as a G2 checkpoint abrogator. These data ensure that the Weel gene signature identified in the present study can be utilized to assess the target engagement level of Wee1 inhibitor in both preclinical and clinical studies.

\section{Results}

Identification of Wee I inhibition signature in cell lines

We previously reported on a novel class of Wee1 inhibitor, MK-1775, (2-alkyl-6-anilino-1-aryl-1, 2-dihydro-3Hpyrazole [3, 4-d]pyrimidin-3-one) with an $\mathrm{IC}_{50}$ value of $5.2 \mathrm{nM}$ against recombinant human Wee1 in in vitro kinase assays. MK-1775 potentiates the anti-cancer efficacy of DNA damaging agents such as gemcitabine, cisplatin, and carboplatin both in vitro and in vivo [16]. In order to find an mRNA gene signature that indicates target engagement of Wee1 inhibitor as a PD biomarker, we analyzed genome-wide expression profiles of p53-positive and -negative isogenic paired cell lines (TOV21G-Vec and TOV21G-shp53) treated with gemcitabine and Wee1 inhibitor. TOV $21 \mathrm{G}$ is an ovarian cancer cell line with wild-type p53 gene. The isogenic pairs of p53-positive and -negative TOV21G cells were generated by transfection with a vector expressing an shRNA targeting p53 or an empty vector, respectively [26]. We employed p53 paired cell lines to find PD markers available in cancer cells independent of p53 status. First, gemcitabine was used to treat the p53 matched pair cell lines for $24 \mathrm{hr}$ to activate S-G2 checkpoints. Next, increasing concentrations of MK-1775 were administered to the cells for $8 \mathrm{hr}$ following the gemcitabine treatment. We confirmed that more significant apoptosis was induced in p53-negative cells compared with p53-positive counterparts in accordance with the previous study [16] (Figure 1). While 28\% and $44 \%$ of the sub-G1 fraction was induced in p53 negative cells treated with $100 \mathrm{nM}$ and $300 \mathrm{nM}$ of the Wee1 inhibitor respectively, $5.9 \%$ and $6.4 \%$ of the sub-G1 fraction was observed in p53-positve cells. In parallel with the efficacy study, mRNA recovered at 8 and $16 \mathrm{hr}$ after the Wee 1 inhibitor treatment was subjected to microarray analysis to find the PD gene biomarker. We extracted genes whose expression levels in Wee1 inhibitor-treated cell lines were significantly up- or down-regulated compared to those of gemcitabine treated cell lines. We pared down the signature by extracting the genes whose expression exhibited greater than three-fold change in both p53 positive and negative cell lines in at least one treatment condition. A hierarchical clustering of the gene signature composed of 55 genes is shown in Figure 2, and the genes exhibited similar expressional regulation in both $\mathrm{p} 53$ positive and negative cells. Moreover, most of the genes showed time-dependent and concentration-dependent expression changes that are suitable features of PD biomarkers. Functional assessment of the gene signature by a hypergeometric test for gene enrichment indicated that S-G2/M cell cycle genes

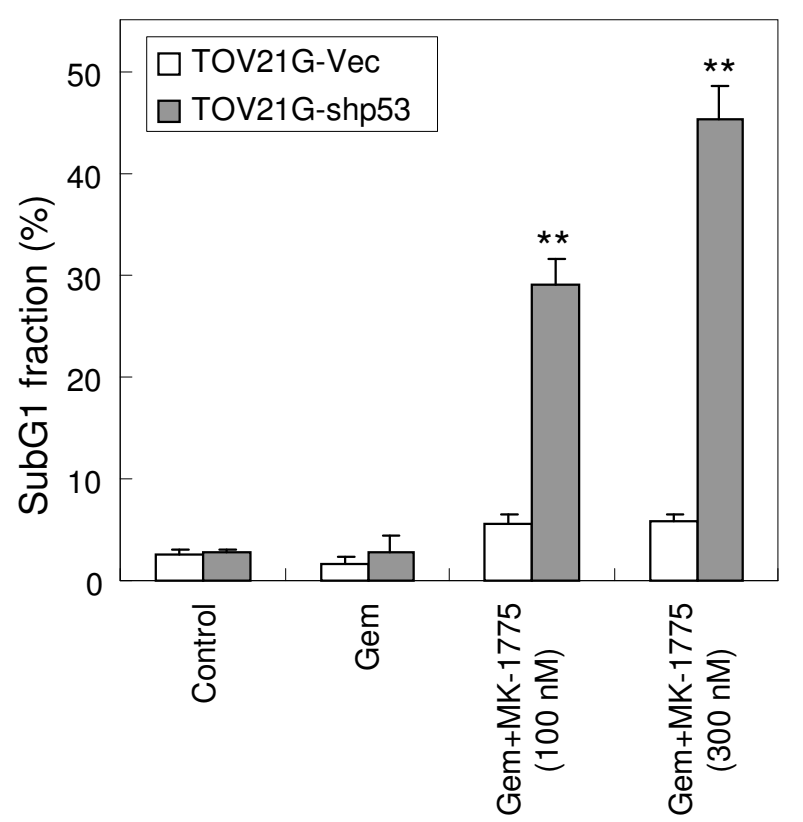

Figure I

TOV2 I G p53 positive and negative matched pair cell lines treated with gemcitabine and Weel inhibitor. TOV2 IG positive- and negative cell lines were treated with $30 \mathrm{nM}$ gemcitabine, and 100 and $300 \mathrm{nM}$ of the Weel inhibitor. At $8 \mathrm{hr}$ post Weel inhibitor treatment, cells were subjected to flow cytometry analysis. The percentage of the subG I fraction corresponds to apoptotic cells. TOV2IGVec: wild-type p53 cells; TOV2IG-shp53: p53-deficient cells; Gem: gemcitabine. **, $P<0.01$, compared with TOV2IGVec.

were significantly enriched in down-regulated genes (DNA replication: $\mathrm{p}=8.4 \times 10^{-9} ; \mathrm{S}$ phase of mitotic cell cycle: $\mathrm{p}=5.3 \times 10^{-8}$; mitotic cell cycle: $\left.\mathrm{p}=1.69 \times 10^{-5}\right)$ and up-regulated genes (nucleosome assembly in $\mathrm{M}$ phase: $\mathrm{p}$ $\left.=1.3 \times 10^{-23}\right)$. This finding is consistent with the function of Wee1 kinase that prevents premature mitosis entry.

\section{Identification of Wee I inhibition signature in rat skin samples}

Although measuring PD biomarkers in tumors is preferable, skin is an attractive tissue since it is easily accessible for analyzing PD effects, especially for tumor types for which biopsies are difficult. In attempting to identify PD biomarkers in surrogate skin tissues in vivo, expression profiles were analyzed between rat skin samples treated with gemcitabine only and a gemcitabine/Wee1 inhibitor combination. Subcutaneous xenograft tumors were formed by injection of the human colorectal cancer, WiDr, in the hind flank of immunodeficient nude rats. On the 8th day, gemcitabine was intraveneously (IV) administrated to the animals. Twenty-four hours later, an 


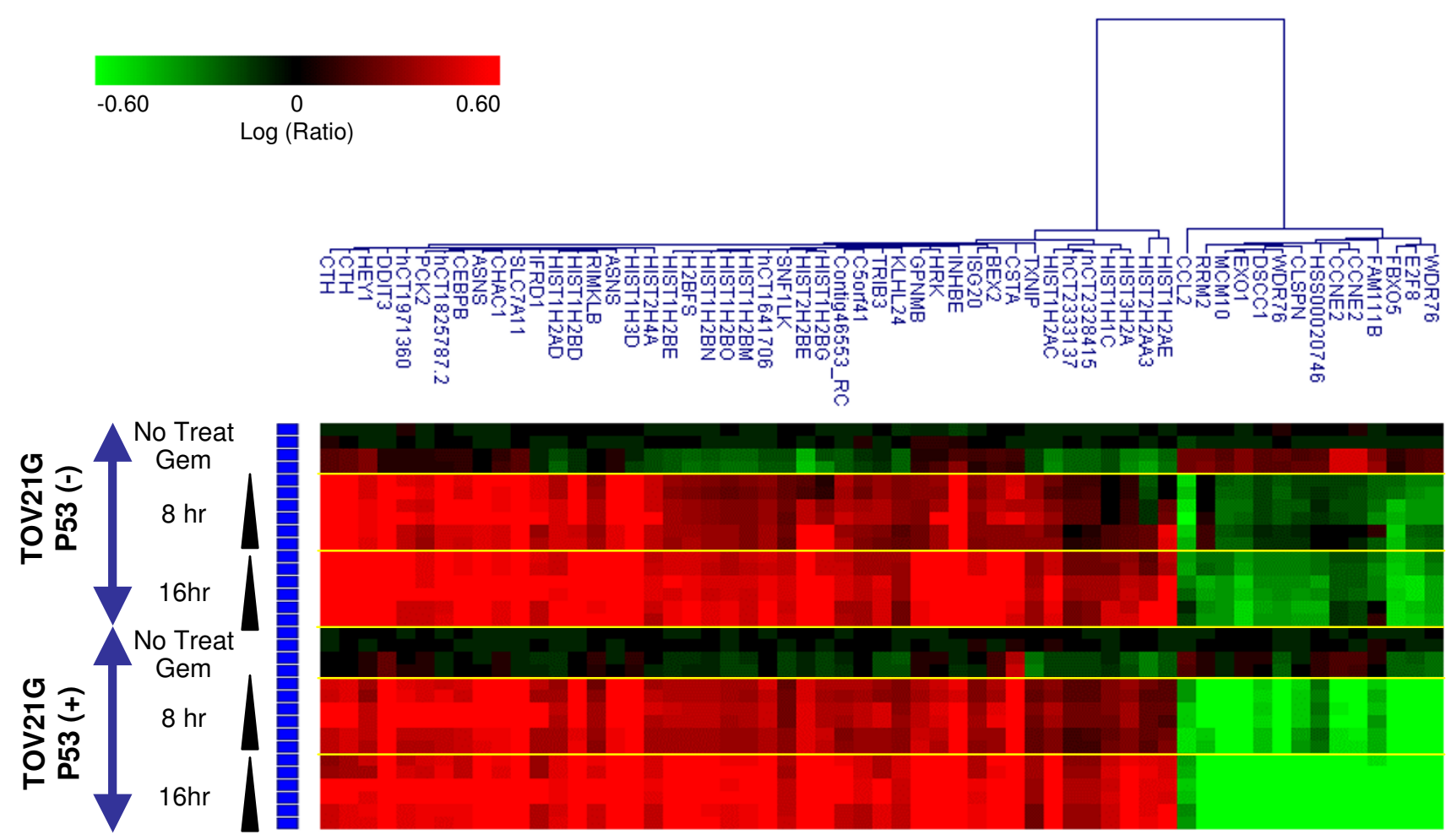

Figure 2

Identification of a gene signature changed by gemcitabine and Weel inhibitor combination in both p53 positive and negative paired cell lines. TOV2 IG positive and negative cell lines were treated with gemcitabine and Wee I inhibitor as described in the legend of Figure I, and mRNA from each treatment sample was applied to microarray analysis. A gene signature whose expression significantly changed in response to gemcitabine and Weel inhibitor treatment was extracted. Each row represents a sample from each treatment group. Each column represents a gene. Red, up-regulated genes; green, down-regulated genes.

increasing concentration of the Weel inhibitor was infused via IV infusion for $8 \mathrm{hr}$. Then, total RNAs from each rat skin tissue were purified and applied to microarray analysis to extract a gene signature whose expression significantly changed in response to gemcitabine and the Wee1 inhibitor treatment. The selection criteria to determine up- and down-regulated genes are described in the Materials and Methods in detail. Briefly, error-weighted ANOVA was applied between the Wee1 inhibitor-treated samples and gemcitabine treated samples, and the genes whose expression changed more than 1.5-fold in either 1.0 or $3.0 \mathrm{mg} / \mathrm{kg} / \mathrm{hr}$ treatment were further selected down. As a result, 48 genes out of 39,558 probes were found to be significantly changed by gemcitabine/Wee1 inhibitor combination treatment compared with gemcitabine treatment only. Hierarchical clustering of the gene signature in rat skin is displayed in Figure 3 as a heatmap, showing the dose-dependent changes in their expressions.

\section{Extraction of Weel inhibition gene signature available in both tumor and skin tissues}

To find genes that can be used as a PD biomarker in both tumor and skin tissues, a common gene signature that was changed in both cancer cell lines and skin tissue was extracted. In both experiments, claspin (CLSPN), minichromosome maintenance complex component 10 (MCM10), and F-box protein 5 (FBXO5) were significantly changed, indicating that they could be promising expression PD biomarkers for the Wee1 inhibitor independent of p53 status and the tissue type. CCNE1 was included in the gene set changed in skin samples, whereas CCNE2 was found in the analysis of p53 paired cell lines in vitro. Given the well-conserved function between CCNE1 and CCNE2, both genes were selected for the Wee1 inhibition gene signature for further validation. Previously reported functions of the five genes in the Wee1 inhibition gene signature which relate to the S-G2 cell cycle are shown in Table 1, inferring a relationship between Wee1 inhibitor-mediated gene expression changes and S-G2 cell cycle checkpoints. 
A

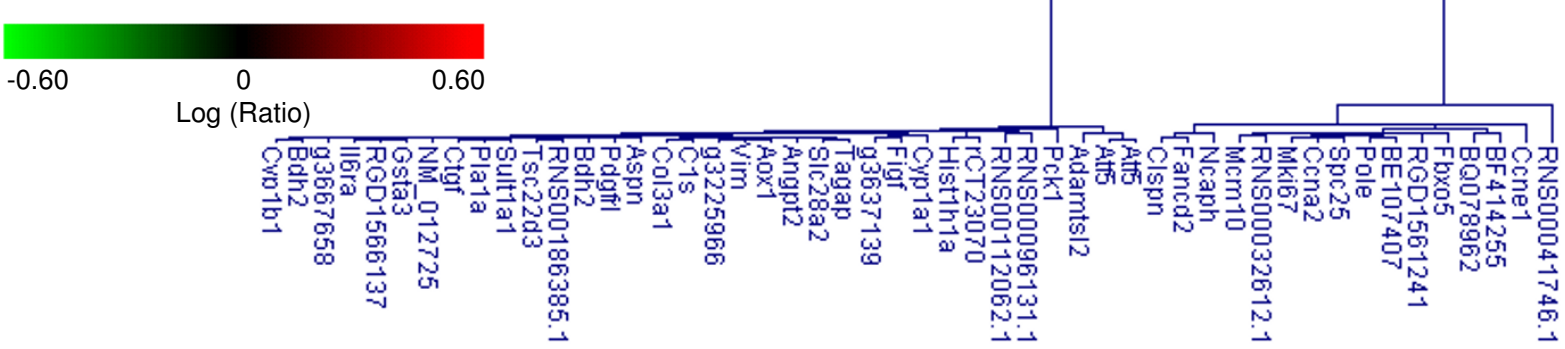

No treat

Gemcitabine

MK-1775 (0.5mg/kg/hr)

MK-1775 (1.0mg/kg/hr)

MK-1775 (3.0mg/kg/hr)

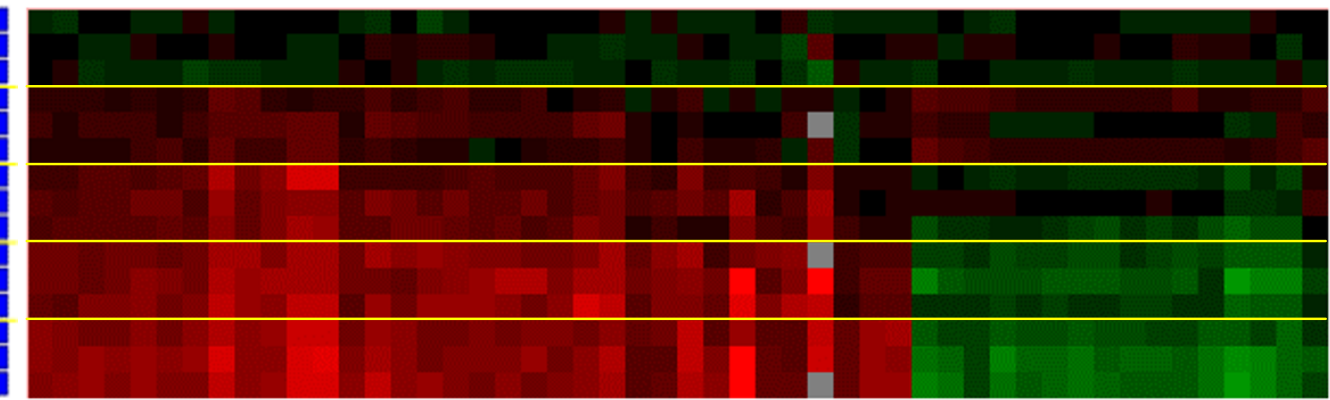

B

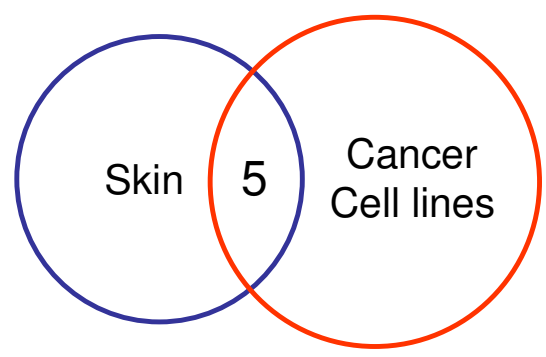

Rat skin administered with

Gem and MK-1775

48 genes

\section{p53 (+) and (-) paired cells with Gem and MK-1775 \\ 55 genes}

Figure 3

Identification of a gene signature changed by gemcitabine and Weel inhibitor in rat skin sample. (A) Expression profile of rat skin samples treated with gemcitabine and Weel inhibitor. Nude rats were administered with gemcitabine and increasing concentrations of the Weel inhibitor via IV infusion. At $8 \mathrm{hr}$ post Weel inhibitor administration, mRNA from each rat skin sample was applied to microarray analysis. A gene signature whose expression significantly changed in response to gemcitabine and Weel inhibitor treatment was extracted. Each row represents a sample from each treatment group. Each column represents a gene. Red, up-regulated genes; green, down-regulated genes. (B) Weel gene signature commonly changed in both cancer cell lines and skin samples. The common signature for both tumor and surrogate tissues was identified by extracting commonly regulated genes in global expression profiling. Gem: gemcitabine; Weeli: Weel inhibitor (MK-1775).

Table I: Function of Weel inhibition gene signature

\begin{tabular}{ll}
\hline Gene & Function \\
\hline CLSPN & Clspn triggers a checkpoint arrest of cell cycle by activating CHEKI in response to DNA damage \\
FBXO5 & Fbxo5 is a mitotic regulator interacting with CDC20 and inhibits the anaphase promoting complex \\
MCMI0 & Involved in S phase progression which interacts with chromatin during S phase and dissociates G2 \\
CCNEI & CyclinEI forms a complex with and functions as a regulatory subunit of CDK2 \\
CCNE2 & CyclinE2 forms a complex with and functions as a regulatory subunit of CDK2
\end{tabular}


Although the 5 genes were selected as a common signature in both cancer and surrogate skin tissues, most of the cancer gene signature and rat skin signature showed statistically significant expression changes in reciprocal experiments, suggesting conserved Wee1-mediated expression changes in both tumor and the surrogate tissues.

\section{Validation of the Wee I inhibition gene signature}

Expression changes of the Wee1 inhibition gene signature in cancer cells have thus far been assessed only in cultured cell lines. To validate the Wee 1 inhibition gene signature, we analyzed mRNA expression of the five genes in WiDr xenograft tumors in vivo. With the same dosing regimen used in the rat skin microarray, nude rats bearing WiDr xenograft tumors were administered with gemcitabine and the Wee1 inhibitor combination. To analyze the gene markers, total RNA samples from the WiDr xenograft tumors were purified $8 \mathrm{hr}$ after Wee1 inhibitor administration, and the expression of the Wee1 gene signature was measured by quantitative RT-PCR. As a result, the expression of all five genes was up-regulated by gemcitabine treatment, and subsequently down-regulated by the Wee 1 inhibitor treatment, which was a similar expression pattern to that of TOV21G p53 matched pair cells in vitro (Figure 4). For example, gemcitabine treatment increased the expression of CLSPN by 2 -fold, and Wee1 inhibitor down-regulated the expression to one-fourth compared with the gemcitabine single treatment sample. We also measured the level of phosphorylated CDC2 (Y15) in the WiDr xenograft tumor samples by Western blotting. The expression pattern of the Weel gene signature was similar to that of phosphorylated-CDC2 (Y15) when the correlation coefficient $(\mathrm{R})$ was calculated between phosphorylated-CDC2 and mRNA expression of each gene in the Wee1 gene signature (Figure 4). This correlation supports the idea that functions of each gene in the Wee1 inhibition signature relate to the S-G2 cell cycle and/or its checkpoints. Regarding anti-tumor efficacy, statistically significant enhancement of efficacy for gemcitabine was observed, when co-treated with more than $0.5 \mathrm{mg} / \mathrm{kg} / \mathrm{hr}$ (8 hr IV infusion) of MK-1775 (data not shown).

Finally, to confirm that the selected genes constitute a genuine Wee1 inhibition signature independent of the inhibition modality, the mRNA expression of the five genes were examined in WiDr cells treated with siRNA for Wee1 in vitro. Twenty-four hours after gemcitabine treatment, siRNA for Wee1 was transferred to the cells and the expression of the candidate signature was analyzed. In accordance with the results obtained in the Wee1 inhibitor study, significant down-regulation of mRNA expression was observed when Wee1 was silenced with siRNA (Figure $5)$.

\section{Discussion}

A number of reports have shown the usefulness of protein biomarkers to assess target engagement of anti-cancer agents in tumors $[20,21,27]$. Some protein markers for the Wee1 inhibitor have also been reported in preclinical studies, including phosphorylated-CDC2 and -histone H3 [14-16]. Assays for protein-markers are in general not quantitative and require large amounts of biopsy specimens in clinical trials. The same holds true for protein markers for the Wee1 inhibitor. The development of a Wee1 gene signature as an mRNA-based expression biomarker offers some advantages over protein markers. The Wee1 gene signature presents quantitative data when measured by RT-PCR. This allows investigators to precisely correlate the changes in the expression of the Wee 1 gene signature and anti-tumor efficacy of the Wee1 inhibitor. The Wee1 gene signature is also superior to conventional IHC markers such as phosphorylated-CDC2 in terms of the required amount of samples. To measure phosphorylated-CDC2 in cancer, several slices of formalin fixed paraffin embedded tissues (FFPET) are required for total CDC2, phosphorylated CDC2, and their confirmation assays. In contrast, one slice will be sufficient for multiple repeated measurements of the Wee1 gene expression signature. Since the quantification and amplification technologies of mRNA have been advancing rapidly $[28,29]$, further reduction of required samples might be possible for analyzing the Wee1 gene signature.

In order to assess accurate target engagement of the Wee 1 inhibitor, it is preferable to measure PD biomarkers in tumors. However, the feasibility of tumor biopsy is dependent on the tumor type [30,31]. While it is relatively easy to obtain tumor biopsies for skin cancers, biopsies of pancreatic or lung cancers are quite difficult. Therefore, the development of biomarkers that are commonly available in both tumors and surrogate tissues is of great benefit. Previous studies have proven that skin biopsies can be used to assess PD biomarkers of anticancer agents as an easily accessible tissue [32,33]. Although the development of mRNA gene expression biomarkers that can be measured in either tumors or surrogate tissues has been reported, the present study is unique in that the identified Wee1 gene signature can be commonly measured in both tumors and surrogate skin tissues. This was achieved by applying genome-wide gene expression profiling in the two tissues and extracting a commonly regulated gene signature. The Wee 1 gene signature in surrogate skin tissues may accelerate the clinical development of the inhibitor by enabling biopsies for most patients at multiple time points.

The Wee1 gene signature is composed of five genes listed in Table 1. Although the method to identify the signature was a non-biased genome-wide approach, the function of 
A
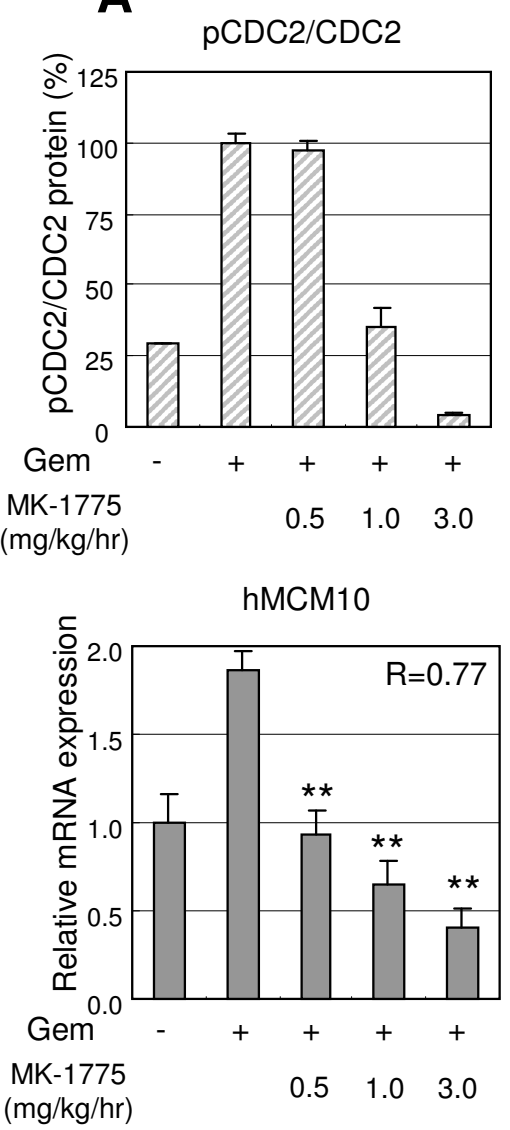

B
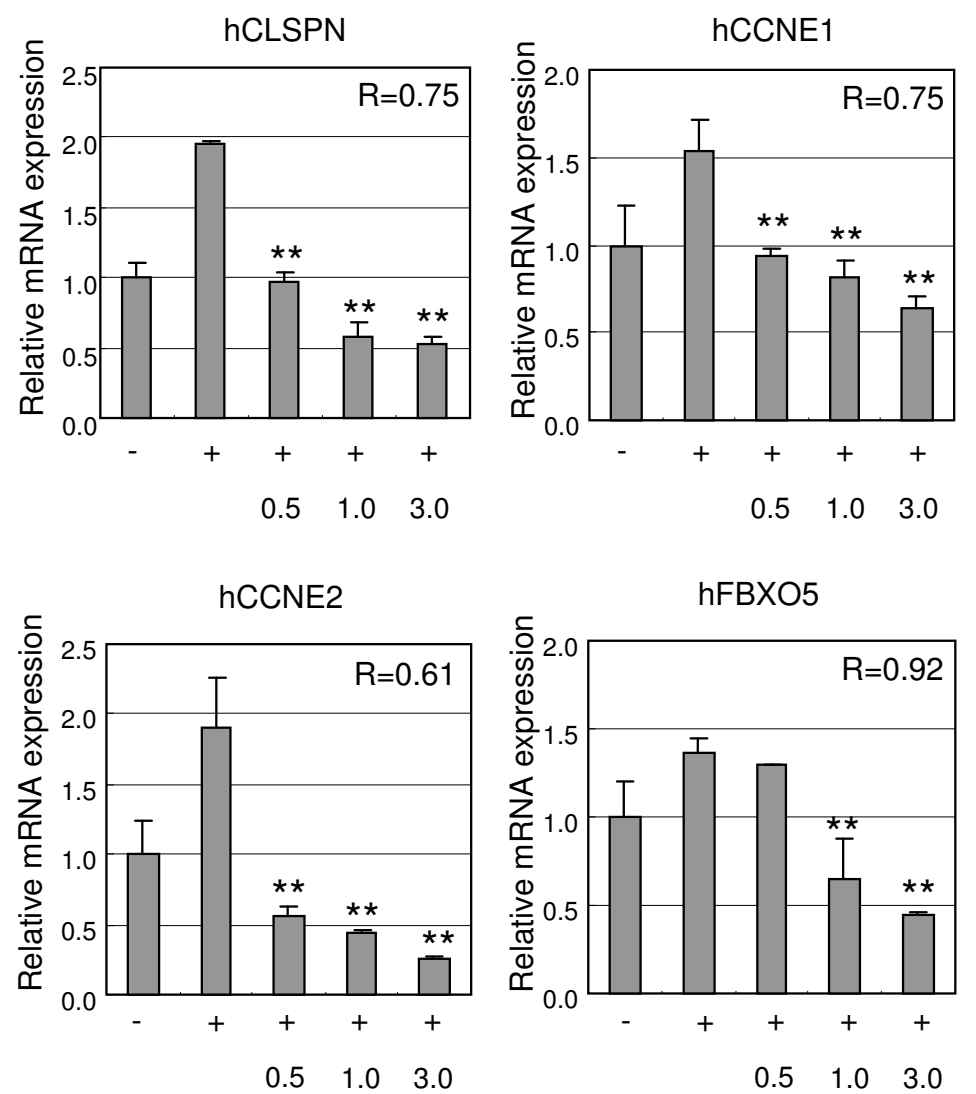

\section{Figure 4}

Expression changes of Weel inhibition signature in xenograft WiDr tumors and their correlation to classical PD marker, pCDC2. Human WiDr colorectal cancer cells were injected subcutaneously into nude rats. After allowing tumors to establish for 8 days, gencitabine and Weel inhibitor were administered in the nude rats as described in the legend of Figure 3. At $8 \mathrm{hr}$ post Weel inhibitor administration, mRNA from each WiDr xenograft tumor was applied to quantitative RTPCR analysis. To correlate the phosphorylated-CDC2 and Weel gene expression signature, the level of the PCDC2 normalized to total $C D C 2$ is shown. The correlation coefficient $(R)$ of $P C D C 2$ expression and each mRNA expression is shown in each graph. Data represent mean $\pm S D$. **, $P<0.01$, compared with gemcitabine treated sample.

each gene in the signature is closely associated with the mechanism underlying the Wee1 inhibitor-mediated SG2 phase checkpoint abrogation. First, CLSPN is a cell cycle regulated protein whose expression peaks at S-G2 phases [34]. CLSPN interacts with CHEK1 kinase that also plays a pivotal role in the S-G2 cell cycle checkpoint, and association of the two proteins is required for CHEK1 activation in response to DNA damage [35]. Therefore, downregulation of CLSPN expression by the Wee1 inhibitor would provide additional beneficial effects on S-G2 checkpoint abrogation by preventing the activation of CHEK1 kinase. Second, MCM10 is a DNA binding protein involved in the initiation of DNA replication as well as the elongation step [36]. Interestingly, it was reported that the depletion of MCM10 by small interfering RNA in cancer cells accumulates DNA damage and arrests the cells in late S-G2 phase, suggesting a role for MCM10 in cell cycle checkpoints [37]. We envision that DNA damage by gemcitabine arrested the cells in the S-G2 phase, which activates the DNA repair system in which MCM10 is involved. The abrogation of the S-G2 phase checkpoint by the Wee1 inhibitor might have reduced the expression of MCM10 without completion of DNA repair. Third, FBXO5, also known as Emi1, is a cellular inhibitor of the APC/C complex which degradates mitotic cyclins (Cyclin A and B) [38]. The up-regulation of FBXO5 ensures that the cells are arrested at $\mathrm{S}$ phase by gemcitabine, since FBXO5 inhibits APC/C during S phases. At the onset of mitosis, it is known that FBXO5 activity is significantly reduced [39], which could also explain the down-regulation of FBXO5 


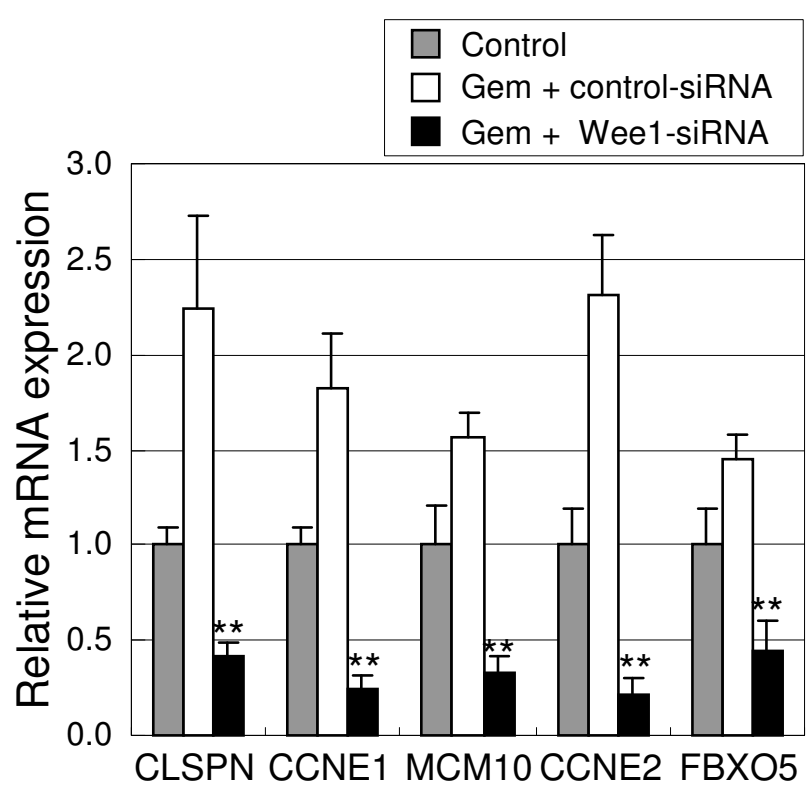

Figure 5

siRNA-Weel treatment confirms that the selected genes are the bona-fide Weel inhibition gene signature. WiDr colorectal cancer cells were treated with $30 \mathrm{nM}$ of gemcitabine and siRNA for Weel. After $48 \mathrm{hr}$ siRNA treatment, mRNA from each was applied to quantitative RTPCR analysis for the Weel gene signature. Gem: gemcitabine. Data represent mean $\pm S D$. $* *, P<0.01$, compared with gemcitabine treated sample.

expression by Wee1 inhibitor. Finally, CyclinE1 and 2 are well-known regulators of $\mathrm{S}$ phase cell cycle progression [40]. Since the expressional regulation of CyclinE has extensively been investigated [41], the expression pattern found in this study was very reasonable. Similar to the hypothetical mechanism discussed for FBXO5, the expression pattern of CyclinE1/2 supports the mode-of-action of the Wee1 inhibitor that causes the disruption of S-G2 checkpoints leading to premature mitotic entry. Although we have speculated a functional relation between the Wee1 inhibitor and the gene signature, it would be interesting to further decipher the molecular role of the five genes in the Wee1 inhibitor-mediated anti-cancer effect.

There are several challenges ahead before using the preclinically developed Wee1 inhibition gene signature in clinical trials. First, although the present data shows that the signature can be assessed as a PD biomarker in surrogate rat skin tissues, the signature should be evaluated in human surrogate tissues. Since the Weel gene signature is composed of cell cycle related genes, their expression changes should be observed in proliferating cells, which is also supported by the fact that actively proliferating tumor samples both in vitro and in vivo showed a larger effect size compared with rat skin tissues. As the actively growing cells in skin samples would be those from hair follicles or hair bulbs, a potential surrogate skin tissue utilized in human clinical trials is scalp punch biopsy, in which hair density is relatively higher compared with other parts of the skin [42]. Plucked hair, including hair follicles and hair bulbs, could be an alternative candidate RNA source for the Wee1 gene signature (Figure 6). It has been reported that plucked hairs can be leveraged as a source of PD markers for other cell cycle inhibitors [43]. Second, the variability of the Weel gene signature is unknown, which makes it difficult to judge whether the observed expression changes in the Weel gene signature are derived from the treatment effect, intrapatient variability, or natural decay of signal. One strategy to address these issues is to conduct phase 0 trials which are first-in-human studies performed before standard phase I trials are conducted [44]. The phase 0 studies may be designed to determine a statistically significant Wee1 inhibitor-mediated effect on the expression changes of the Weel gene signature. With the data from multiple time points both pre- and posttreatment with Wee1 inhibitor, the phase 0 study will provide us with variability data which will allow researchers to do a statistical power calculation for the PD effect for a future standard phase I study.

Despites several challenges for the future of the Wee1 gene signature, its assessment will have beneficial impacts on the development of the Wee1 inhibitor. The quantitative assessment of the signature will allow us to make early decisions even at dose-setting phase 1 trials by providing information on whether sufficient target engagement is achieved or not at tolerable doses.

\section{Conclusion}

In this study, we identified a Weel gene signature whose expression was changed in response to a combination treatment of gemcitabine and Wee1 inhibitor. A common expressional regulation of the Wee1 gene signature was observed in xenograft tumor (p53 negative), cultured cancer cells ( $\mathrm{p} 53$ positive and negative), and rat skin tissues (p53 positive). Although the signature was selected through genome-wide molecular expression, the functions of the genes are associated with S-G2 cell cycle checkpoint and their abrogation, which is also supported by the fact that the phosphorylated CDC2 level that represents the S-G2 checkpoint activation level is highly correlated with the expression pattern of the Wee1 signature genes. In addition to the common regulation of the signature genes independent of the tissue type and p53 status, Wee1-silencing by siRNA confirmed that the Wee1 gene signature is generally regulated by gemcitabine and Wee1 inhibition. The present study first found and validated the gene signature as a PD biomarker for Wee1 inhibitor, and also presented initial evidence that a common mRNA 


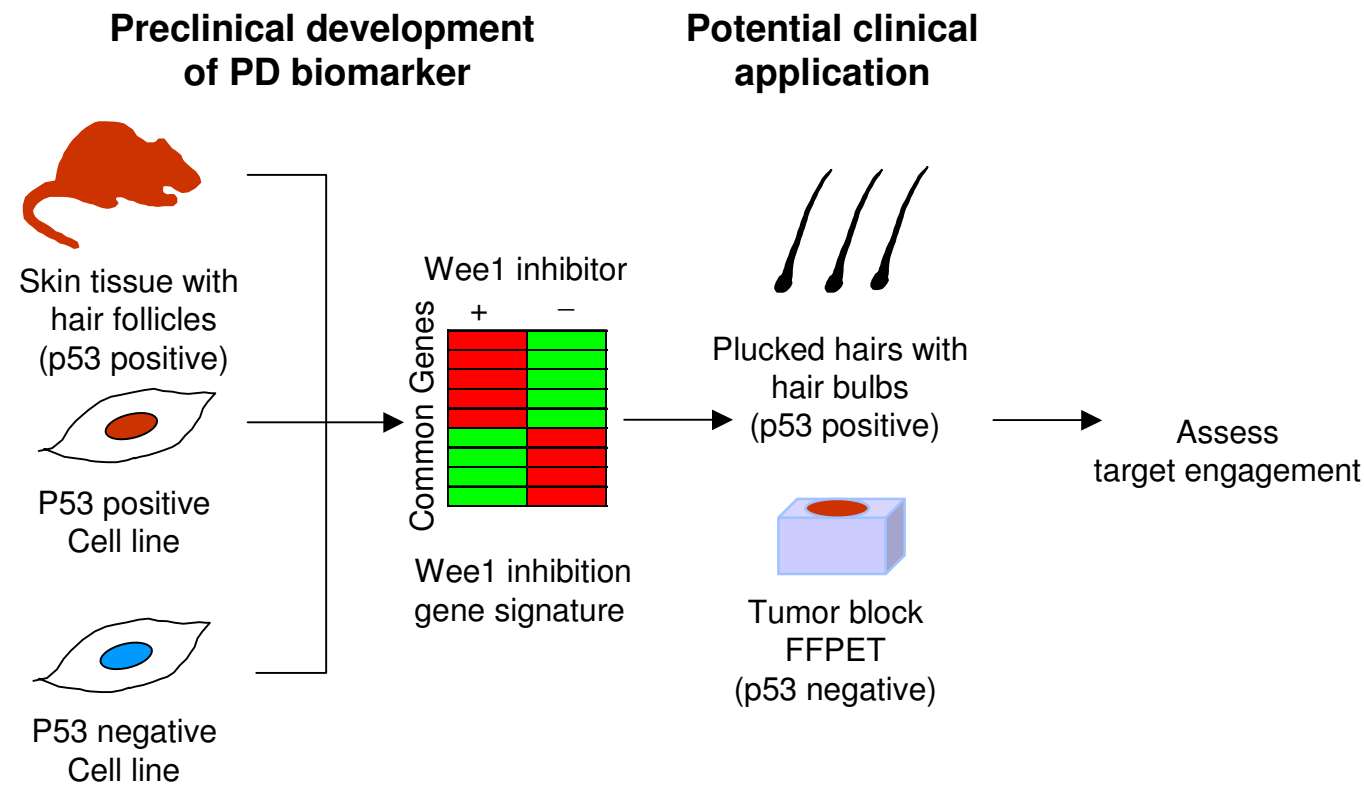

\section{Figure 6}

Schematic diagram for finding the Weel gene signature available for both tumor and surrogate tissues, and its potential application to clinical research. The present study isolated a gene set that commonly showed expression changes in both TOV2 IG p53 positive and negative matched pair cell lines and rat skin tissues, which led to the identification of the Weel gene signature available as a PD biomarker for Weel inhibitor independent of the p53 status of the tissues analyzed. In future clinical trials, the Weel gene expression signature can be analyzed from both plucked hair samples and FFPE tumor samples to assess the target engagement of the Weel inhibitor.

expression-based biomarker in tumors and surrogate tissues can be identified, which is an advantageous feature to facilitate anticancer drug development.

\section{Methods}

\section{Cell culture}

WiDr cell lines were obtained from the American Type Culture Collection, and were cultured according to the supplier's instructions. TOV21G p53-isogenic matchedpair cell lines were provided from ROSETTA INPHARMATICS [26], and were cultured with Dulbecco's Modified Eagle Medium (DMEM, Invitrogen).

\section{Flow cytometric analysis}

Cells were first treated with $30 \mathrm{nM}$ gemcitabine (GEMZER, Lilly) for $24 \mathrm{hr}$ followed by addition of MK1775 for $8 \mathrm{hr}$. Trypsinized single-cells were stained with propidium iodide with the CycleTEST plus DNA reagent kit (BD biosciences) and were analyzed in a FACS Calibur (Becton Dickinson) apparatus.

\section{Expression profiling of TOV2 IG p53 positive- and negative-matched pair cell lines}

TOV-21G p53-isogenic matched-pair cell lines were treated with $30 \mathrm{nM}$ gemcitabine for 24-hr, followed by addition of MK-1775. At 8-hr or 16-hr after MK-1775 treatment, cells were recovered for RNA extraction. Hybridization for microarray experiments was performed as follows: TOV21G-Vec, no treatment control (control) vs. TOV21G-Vec. No treatment $(\mathrm{n}=2)$; Control vs. TOV$21 \mathrm{G}-V e c$ treated with $30 \mathrm{nM}$ gemcitabine for $24 \mathrm{hr}(\mathrm{n}=2)$; Control vs. TOV21G-Vec treated with $30 \mathrm{nM}$ gemcitabine for $24 \mathrm{hr}$, followed by treatment with $100 \mathrm{nM}, 300 \mathrm{nM}$, or $1000 \mathrm{nM}$ of MK-1775 for $8 \mathrm{hr}(\mathrm{n}=2$ for each concentration of MK-1775); Control vs. TOV21G-Vec treated with $30 \mathrm{nM}$ gemcitabine for $24 \mathrm{hr}$, followed by treatment with $100 \mathrm{nM}, 300 \mathrm{nM}$ or $1000 \mathrm{nM}$ of MK-1775 for $16 \mathrm{hr}(\mathrm{n}=$ 2 for each concentration of MK-1775). The same hybridizations performed for TOV21G-Vec were also carried out for the TOV21G-shp53 cell line.

\section{Gene marker findings of in vivo WiDr xenograft nude rats} The PD gene biomarker was investigated in vivo in a WiDr nude rat xenograft model. Gemcitabine was dosed as an intravenous bolus ( $50 \mathrm{mg} / \mathrm{kg}$ ). After $24 \mathrm{hr}$ of gemcitabine administration, MK-1775 was dosed via intravenous infusion at doses of $0.5,1.0$, and $3.0 \mathrm{mg} / \mathrm{kg} / \mathrm{hr}$ for $8 \mathrm{hr}$. Skin samples were isolated $8 \mathrm{hr}$ after MK-1775 dosing. Hybridization for microarray experiments was performed as follows: Vehicle control pool (control) vs. Vehicle control self-reference $(\mathrm{n}=3)$; Control vs. gemcitabine $50 \mathrm{mg} / \mathrm{kg}$ $(\mathrm{n}=3)$; Control vs. gemcitabine $50 \mathrm{mg} / \mathrm{kg}$ with $0.5,1.0$, 
or $3.0 \mathrm{mg} / \mathrm{kg} / \mathrm{hr}$ of MK-1775 for $8 \mathrm{hr}$ ( $\mathrm{n}=3$ for each concentration of MK-11775).

Total RNA from cultured cells or skin samples was isolated by using the RNeasy mini kit (Qiagen, \#74104) with DNase I (Qiagen, \#79254). Total RNA from skin or tumor tissues in rat xenograft model was isolated by Trizol reagent (Invitrogen, \#15596-018), and the isolated RNA was repurified with an RNeasy mini kit. The purified RNA from each sample was converted to cDNA and hybridized to appropriate reference standards; rat skin microarray: three vehicle control samples; human cell line microarray: pooled TOV21G with control vector samples. Next, microarray analysis was performed with a Rosetta/Merck microarray, Human $44 \mathrm{k} 1.1$ and Rat $44 \mathrm{k} \mathrm{1.1.} \mathrm{Expression}$ profiles were analyzed by the microarray software, Resolver (Rosetta Inpharmatics) to identify the classifier genes for responder.

\section{Microarray data analysis}

1) Rat skin sample: First, error-weighted ANOVA was applied between 1.0/3.0 mg/kg/hr MK-1775 treated samples and gemcitabine only treated samples, and the genes whose expression was significantly changed in both 1.0 and 3.0 mpk treatment ( $\mathrm{p} \leq 0.001$ ) were extracted. Next, we selected genes whose expression changed more than 1.5 -fold in either 1.0 or $3.0 \mathrm{mg} / \mathrm{kg} / \mathrm{hr}$ treatment compared with gemcitabine only treated samples. Then, errorweighted ANOVA was applied between $3.0 \mathrm{mg} / \mathrm{kg} / \mathrm{hr} \mathrm{MK}-$ 1775 treated samples and 0.5 mpk MK-1775 treated samples, and the genes whose expression significantly ( $\mathrm{p} \leq$ 0.05 ) changed were selected.

2) TOV21G-derived p53 matched pair cells: In each experiment of TOV21 p53 positive and negative cell lines, expression levels of MK-1775 treated cell lines were divided by those of untreated cell lines with the re-ratio algorithm in Resolver. (Calculated values were used for clustering). In each experiment of TOV21 p53 positive and negative cell lines, gene expression of MK-1775 treated cell lines were divided by those of only gemcitabine treated cell lines with the re-ratio algorithm in Resolver. (Calculated values were used for signature selection). After the re-ratio, signature genes, whose expression levels in MK-1775 treated cell lines were significantly upor down-regulated compared to those of gemcitabine treated cell lines ( $\mathrm{p} \leq 0.01)$, were selected in all comparisons. Among the signatures, we further selected genes which exhibited greater than three-fold expression change in at least one condition in both vector and control samples.

For each set of the selected signatures, hierarchical clustering was done by the Rosetta Resolver system with cosine correlation and average link options.

\section{Quantitative RT-PCR Analysis}

cDNA was synthesized from $1 \mu \mathrm{g}$ of total RNA by using TaqMan reverse transcription reagents (PE Applied Biosystems, \#N8080234). Quantitative real-time PCR assays for human CLSPN, CCNE1/2, MCM10, FBXO5, and GAPDH were performed in triplicate for cDNA samples in 96-well optical plates. Data were collected and analyzed using an ABI PRISM 7700 sequence detector system (PE Applied Biosystems). Primer and probe sequences for the quantitative RT-PCR are as follows: primers for CLSPN, 5'AGGTGGAGGAAGGAGCGAA-3', 5'-TTTCCCCTGCTGTGCCAT-3'; Taqman probe for CLSPN, 5'-TGAACGAGAGCAGTGGCTTCGGG-3'; primers for CCNE1, 5'AAATGGCCAAAATCGACAGG-3', 5'-TGCATTATTGTCCCAAGGCTG-3'; Taqman probe for CCNE1: 5'CGGCGAGGGACCAGTGTGGG-3'; primers for CCNE2 by SYBR Green method, 5'-CTATTTGGCTATGCTGGAGGAAGT 3', 5'-TTCAGTGCTCTTCGGTGGTGT-3'; primers for MCM10 by SYBR Green method, 5'-CTCCAGATCCCAAAAGCTCATC-3', 5'-TGTTCCGAGAAATCGTCTGTAGG-3'; primers for FBXO5 by SYBR Green method, 5'TGAAGAAGGTAGCCTCCTGGAG-3', 5'-TGGCAGCAAGTTTTTGTTGG-3'.

\section{Phosphorylated-CDC2 assay}

Tumors were isolated $8 \mathrm{hr}$ after MK-1775 dosing. CDC2 protein was solubilized by homogenizing cells in buffer containing $1 \%$ NP40, $0.1 \%$ Triton X-100, and was detected by Western blotting with an anti-p-CDC2Y15 specific antibody (Cell Signaling \#9111). The captured antibodies were detected and stained with biotinylated anti-IgG and streptavidin/horse radish peroxidase. The immunostained area was quantified using Image Pro Plus software (Media Cybertics Inc.).

\section{Competing interests}

The authors declare that they have no competing interests.

\section{Authors' contributions}

SM conducted most of the experiments, performed data analysis, and drafted the manuscript. KY and HI performed microarray data analysis. TA performed the in vivo study, and TN performed the in vitro RT-PCR expression study. HH contributed to the design of the in vivo experiments and edited the manuscript. HK contributed to the entire design of the experiments. All authors read and approved the final manuscript.

\section{References}

I. Yao SL, Akhtar AJ, McKenna KA, Bedi GC, Sidransky D, Mabry M, Ravi R, Collector MI, Jones RJ, Sharkis SJ, Fuchs EJ, Bedi A: Selective radiosensitization of $\mathrm{p} 53$-deficient cells by caffeine-mediated activation of p34cdc2 kinase. Nat Med 1996, 2: I | 40- I I 43.

2. Allday MJ, Inman GJ, Crawford DH, Farrell PJ: DNA damage in human B cells can induce apoptosis, proceeding from GI/S when p53 is transactivation competent and G2/M when it is transactivation defective. EMBO J 1995, 14:4994-5005. 
3. Zabludoff SD, Deng C, Grondine MR, Sheehy AM, Ashwell S, Caleb BL, Green S, Haye HR, Horn CL, Janetka JW, Liu D, Mouchet E, Ready S, Rosenthal JL, Queva C, Schwartz GK, Taylor KJ, Tse AN, Walker GE, White AM: AZD a novel checkpoint kinase inhibitor, drives checkpoint abrogation and potentiates DNA-targeted therapies. Mol Cancer Ther 7762, 7:2955-2966.

4. Hirao A, Kong YY, Matsuoka S, Wakeham A, Ruland J, Yoshida H, Liu $D$, Elledge SJ, Mak TW: DNA damage-induced activation of p53 by the checkpoint kinase Chk2. Science 2000, 287:|824-|827.

5. Dasika GK, Lin SC, Zhao S, Sung P, Tomkinson A, Lee EY: DNA damage-induced cell cycle checkpoints and DNA strand break repair in development and tumorigenesis. Oncogene 1999, 18:7883-7899.

6. Goi K, Takagi M, Iwata S, Delia D, Asada M, Donghi R, Tsunematsu $Y$, Nakazawa S, Yamamoto H, Yokota J, Tamura K, Saeki Y, Utsunomiya J, Takahashi T, Ueda R, Ishioka C, Eguchi M, Kamata N, Mizutani S: DNA damage-associated dysregulation of the cell cycle and apoptosis control in cells with germ-line p53 mutation. Cancer Res 1997, 57:1895-1902.

7. Mizuarai S, Yamanaka K, Kotani H: Mutant p53 induces the GEF$\mathrm{HI}$ oncogene, a guanine nucleotide exchange factor- $\mathrm{HI}$ for RhoA, resulting in accelerated cell proliferation in tumor cells. Cancer Res 2006, 66:6319-6326.

8. Lundgren K, Walworth N, Booher R, Dembski M, Kirschner M, Beach $D$ : mik $I$ and weel cooperate in the inhibitory tyrosine phosphorylation of cdc2. Cell I99I, 64: IIII-II22.

9. Rowley R, Hudson J, Young PG: The weel protein kinase is required for radiation-induced mitotic delay. Nature 1992, 356:353-355.

10. Stanford JS, Ruderman JV: Changes in regulatory phosphorylation of Cdc25C Ser287 and Weel Ser549 during normal cell cycle progression and checkpoint arrests. Mol Biol Cell 2005, 16:5749-5760

II. Lee J, Kumagai A, Dunphy WG: Positive regulation of Wee I by ChkI and 14-3-3 proteins. Mol Biol Cell 200I, I 2:55I-563.

12. Rothblum-Oviatt CJ, Ryan CE, Piwnica-Worms H: 14-3-3 binding regulates catalytic activity of human Wee I kinase. Cell Growth Differ 200I, I 2:58I-589.

13. Wang Y, Li J, Booher RN, Kraker A, Lawrence T, Leopold WR, Sun $Y$ : Radiosensitization of $\mathrm{p} 53$ mutant cells by PD0 16 a novel G(2) checkpoint abrogator. Cancer Res 6285, 61:82II-82 I7.

14. Li J, Wang Y, Sun Y, Lawrence TS: Wild-type TP53 inhibits G(2)phase checkpoint abrogation and radiosensitization induced by PDOI6 a WEEI kinase inhibitor. Radiat Res. 6285, I 57(3):322-330.

15. Wang Y, Decker SJ, Sebolt-Leopold J: Knockdown of Chk I, Wee I and Myt I by RNA interference abrogates $G 2$ checkpoint and induces apoptosis. Cancer Biol Ther 2004, 3:305-3/3.

16. Hirai H, Iwasawa Y, Okada M, Arai T, Nishibata T, Kobayashi M, Kimura T, Kaneko N, Ohtani J, Sakai T, Mizuarai S, Yoshizumi T, Hidehito Kotani: Inhibition of Weel kinase by small molecule inhibitor enhances anti-tumor efficacy of DNA damaging agents specifically in p53-negative cancers. Proceedings of the 100th Annual Meeting of the American Association for Cancer Research 2009:3732.

17. Sarker D, Workman P: Pharmacodynamic biomarkers for molecular cancer therapeutics. Adv Cancer Res 2007, 96:213-268.

18. Cummings J, Ward TH, Greystoke A, Ranson M, Dive C: Biomarker method validation in anticancer drug development. $\mathrm{Br} J \mathrm{Phar}$ macol 2008, I 53:646-656.

19. Workman P, Aboagye EO, Chung YL, Griffiths JR, Hart R, Leach MO, Maxwell RJ, McSheehy PM, Price PM, Zweit J, Cancer Research UK: Pharmacodynamic/Pharmacokinetic Technologies Advisory Committee. Minimally invasive pharmacokinetic and pharmacodynamic technologies in hypothesis-testing clinical trials of innovative therapies. I Natl Cancer Inst 2006, 98:580-598.

20. Albanell J, Rojo F, Averbuch S, Feyereislova A, Mascaro JM, Herbst R, LoRusso P, Rischin D, Sauleda S, Gee J, Nicholson RI, Baselga J: Pharmacodynamic studies of the epidermal growth factor receptor inhibitor ZDI839 in skin from cancer patients: histopathologic and molecular consequences of receptor inhibition. J Clin Oncol 2002, 20: I I0-124.

21. White D, Saunders V, Lyons AB, Branford S, Grigg A, To LB, Hughes $T$ : In vitro sensitivity to imatinib-induced inhibition of $A B L$ kinase activity is predictive of molecular response in patients with de novo CML. Blood 2005, 106:2520-2526.

22. Jhala N, Jhala D, Vickers SM, Eltoum I, Batra SK, Manne U, Eloubeidi $\mathrm{M}$, Jones JJ, Grizzle WE: Biomarkers in Diagnosis of pancreatic carcinoma in fine-needle aspirates. Am J Clin Pathol 2006, 1 26:572-579.

23. Hardwick JS, Yang Y, Zhang C, Shi B, McFall R, Koury EJ, Hill SL, Dai $H$, Wasserman R, Phillips RL, Weinstein EJ, Kohl NE, Severino ME, Lamb JR, Sepp-Lorenzino L: Identification of biomarkers for tumor endothelial cell proliferation through gene expression profiling. Mol Cancer Ther 2005, 4:4|3-425.

24. Soo RA, Wu J, Aggarwal A, Tao Q, Hsieh W, Putti T, Tan KB, Low JS, Lai YF, Mow B, Hsu S, Loh KS, Tan L, Tan P, Goh BC: Celecoxib reduces microvessel density in patients treated with nasopharyngeal carcinoma and induces changes in gene expression. Ann Oncol 2006, I 7:1625-1630.

25. Beliën A, De Schepper S, Floren W, Janssens B, Mariën A, King P, Van Dun J, Andries L, Voeten J, Bijnens L, Janicot M, Arts J: Real-time gene expression analysis in human xenografts for evaluation of histone deacetylase inhibitors. Mol Cancer Ther 2006, 5:2317-2323.

26. Bartz SR, Zhang Z, Burchard J, Imakura M, Martin M, Palmieri A, Needham R, Guo J, Gordon M, Chung N, Warrener P, Jackson AL, Carleton M, Oatley M, Locco L, Santini F, Smith T, Kunapuli P, Ferrer M, Strulovici B, Friend SH, Linsley PS: Small interfering RNA screens reveal enhanced cisplatin cytotoxicity in tumor cells having both BRCA network and TP53 disruptions. Mol Cell Biol 2006, 26:9377-9386.

27. Williams R, Baker AF, Ihle NT, Winkler AR, Kirkpatrick L, Powis G: The skin and hair as surrogate tissues for measuring the target effect of inhibitors of phosphoinositide-3-kinase signaling. Cancer Chemother Pharmacol 2006, 58:444-450.

28. Kurimoto K, Yabuta $Y$, Ohinata $Y$, Saitou M: Global single-cell cDNA amplification to provide a template for representative high-density oligonucleotide microarray analysis. Nat Protoc 2007, 2:739-752.

29. Zheng Z, Luo Y, McMaster GK: Sensitive and quantitative measurement of gene expression directly from a small amount of whole blood. Clin Chem 2006, 52:1294-1302.

30. Dowlati A, Haaga J, Remick SC, Spiro TP, Gerson SL, Liu L, Berger SJ, Berger NA, Willson JK: Sequential tumor biopsies in early phase clinical trials of anticancer agents for pharmacodynamic evaluation. Clin Cancer Res 200I, 7:297I-2976.

31. Florentine BD, Cobb CJ, Frankel K, Greaves T, Martin SE: Core needle biopsy. A useful adjunct to fine-needle aspiration in select patients with palpable breast lesions. Cancer 1997, 81:33-39.

32. Vanhoefer U, Tewes M, Rojo F, Dirsch O, Schleucher N, Rosen O, Tillner J, Kovar A, Braun AH, Trarbach T, Seeber S, Harstrick A, Baselga J: Phase I study of the humanized antiepidermal growth factor receptor monoclonal antibody EMD72000 in patients with advanced solid tumors that express the epidermal growth factor receptor. J Clin Oncol 2004, 22: I 75- I84.

33. Malik SN, Siu LL, Rowinsky EK, deGraffenried L, Hammond LA, Rizzo J, Bacus S, Brattain MG, Kreisberg Jl, Hidalgo M: Pharmacodynamic evaluation of the epidermal growth factor receptor inhibitor OSI-774 in human epidermis of cancer patients. Clin Cancer Res 2003, 9:2478-2486.

34. Chini CC, Chen J: Human claspin is required for replication checkpoint control. J Biol Chem 2003, 278:30057-30062.

35. Kumagai A, Dunphy WG: Claspin, a novel protein required for the activation of Chkl during a DNA replication checkpoint response in Xenopus egg extracts. Mol Cell 2000, 6:839-849.

36. Ricke RM, Bielinsky AK: Mcm I 0 regulates the stability and chromatin association of DNA polymerase-alpha. Mol Cell 2004, 16:173-185.

37. Chattopadhyay S, Bielinsky AK: Human Mcm IO regulates the catalytic subunit of DNA polymerase-alpha and prevents DNA damage during replication. Mol Biol Cell 2007, I 8:4085-4095.

38. Machida YJ, Dutta A: The APC/C inhibitor, Emil, is essential for prevention of rereplication. Genes Dev 2007, 21 : I 84-194.

39. Moshe Y, Boulaire J, Pagano M, Hershko A: Role of Polo-like kinase in the degradation of early mitotic inhibitor $I$, a regulator of the anaphase promoting complex/cyclosome. Proc Natl Acad Sci USA 2004, I 01 :7937-7942. 
40. Hinchcliffe EH, Li C, Thompson EA, Maller JL, Sluder G: Requirement of Cdk2-cyclin $E$ activity for repeated centrosome reproduction in Xenopus egg extracts. Science 1999, 283:85I-854.

41. Dulić V, Lees E, Reed SI: Association of human cyclin E with a periodic GI-S phase protein kinase. Science 1992, 257:1958-196I.

42. Saitoh M, Uzuka M, Sakamoto M: Human hair cycle. J Invest Dermatol 1970, 54:65-8I.

43. Camidge DR, Pemberton M, Growcott J, Amakye D, Wilson D, Swaisland H, Forder C, Wilkinson R, Byth K, Hughes A: A phase I pharmacodynamic study of the effects of the cyclin-dependent kinase-inhibitor AZD5438 on cell cycle markers within the buccal mucosa, plucked scalp hairs and peripheral blood mononucleocytes of healthy male volunteers. Cancer Chemother Pharmacol 2007, 60:479-488.

44. Murgo AJ, Kummar S, Rubinstein L, Gutierrez M, Collins J, Kinders R, Parchment RE, Ji J, Steinberg SM, Yang SX, Hollingshead M, Chen A, Helman L, Wiltrout R, Tomaszewski JE, Doroshow JH: Designing phase 0 cancer clinical trials. Clin Cancer Res 2008, I 4:3675-3682.

Publish with Bio Med Central and every scientist can read your work free of charge

"BioMed Central will be the most significant development for disseminating the results of biomedical research in our lifetime. "

Sir Paul Nurse, Cancer Research UK

Your research papers will be:

- available free of charge to the entire biomedical community

- peer reviewed and published immediately upon acceptance

- cited in PubMed and archived on PubMed Central

- yours - you keep the copyright

Submit your manuscript here:

http://www.biomedcentral.com/info/publishing_adv.asp
BioMedcentral 